\title{
ZIKV viral proteins and their roles in virus-host interactions
}

\author{
Moujian Guo ${ }^{1}$, Lixia Hui ${ }^{1}$, Yiwen $\mathrm{Nie}^{1}$, Boris Tefsen $^{2} \&$ Ying $\mathrm{Wu}^{1,3^{*}}$ \\ ${ }^{1}$ State Key Laboratory of Virology, School of Basic Medical Sciences, Wuhan University, Wuhan 430071, China; \\ ${ }^{2}$ Department of Biological Sciences, Xi'an Jiaotong-Liverpool University, Suzhou 215123, China; \\ ${ }^{3}$ Hubei Province Key Laboratory of Allergy and Immunology, Wuhan 430071, China
}

Received June 6, 2020; accepted September 12, 2020; published online October 14, 2020

\begin{abstract}
The re-emergence of Zika virus (ZIKV) and its associated neonatal microcephaly and Guillain-Barré syndrome have led the World Health Organization to declare a global health emergency. Until today, many related studies have successively reported the role of various viral proteins of ZIKV in the process of ZIKV infection and pathogenicity. These studies have provided significant insights for the treatment and prevention of ZIKV infection. Here we review the current research advances in the functional characterization of the interactions between each ZIKV viral protein and its host factors.
\end{abstract}

ZIKV, viral protein, host-virus interactions, immune response, pathogenicity

Citation: Guo, M., Hui, L., Nie, Y., Tefsen, B., and Wu, Y. (2021). ZIKV viral proteins and their roles in virus-host interactions. Sci China Life Sci 64, 709-719. https://doi.org/10.1007/s11427-020-1818-4

\section{Introduction}

Zika virus (ZIKV) is a mosquito-borne flavivirus that can cause Zika Fever, the Guillain-Barré syndrome (GBS) in adults and microcephaly in fetuses and infants (Johansson et al., 2016; Pierson and Diamond, 2018). ZIKV was first isolated from a rhesus monkey with fever in Zika Forest, Entebbe, Uganda, in 1947 (Dick and Haddow, 1952; Dick et al., 1952). At the outset, ZIKV was only circulating in Africa, and fewer than 20 human infections had been documented for half a century (Faye et al., 2014). In 2007, the first outbreak of ZIKV occurred on Yap Island in the Western Pacific Micronesia, causing approximately 3/4 of the residents to be infected with ZIKV, and the patients had only mild symptoms such as rash, fever, joint pain and conjunctivitis (Duffy et al., 2009; Musso and Gubler, 2016). From October 2013 to March 2014, French Polynesia experienced a large-scale Zika virus infection. Unlike the past, in this epidemic there were cases of GBS reported to be

*Corresponding author (email: yingwu@whu.edu.cn) related with ZIKV infection for the first time (Cao-Lormeau et al., 2014; Oehler et al., 2014). The largest outbreak of ZIKV infection in history started in Brazil in 2015, and subsequently the virus infections rapidly expanded to many countries in the Americas (Fauci and Morens, 2016). As of 2017 , more than 22,000 confirmed cases and 580,000 suspected cases have been reported in 52 American countries and regions (Pierson and Diamond, 2018). Due to the neonatal microcephaly and GBS associated with ZIKV infections in the American ZIKV epidemic, ZIKV received widespread international attention (de Oliveira et al., 2017; Johansson et al., 2016).

ZIKV belongs to the genus of Flavivirus in the family of Flaviviridae. The virus particle is about $40 \mathrm{~nm}$ in diameter and comprises an icosahedral shell consisting of 180 copies each of the envelope (E) protein and the membrane (M) protein, which are all inserted into the lipid membrane with their transmembrane domains (Hamel et al., 2015; Shi and Gao, 2017). The ZIKV genome is a positive-sense, singlestranded RNA of approximately $11 \mathrm{~kb}$ in length, and contains two untranslated regions (UTR) and one open reading frame 
(ORF) with 10 genes (Shi and Gao, 2017). The translation of the ORF produces a polyprotein of more than 3,000 amino acid residues which is cleaved by the host and viral proteases to produce 10 viral proteins including three structural proteins (capsid protein (C), pre-membrane (prM) and envelope protein (E)), and seven nonstructural proteins (NS1, NS2A, NS2B, NS3, NS4A, NS4B and NS5) (Hasan et al., 2018; Shi and Gao, 2017). The structural proteins are mainly involved in the assembly of virus particles, virus adsorption and invasion into cells, and contain the major epitopes of virus. The non-structural proteins are mainly responsible for viral genome replication, translation and regulation of the host immune responses and metabolism. ZIKV is mainly transmitted through several mosquito species of the genus Aedes, such as Aedes aegypti, Aedes albopictus, Aedes Hensili, Aedes polynesiensis, Aedes africanus and Aedes luteocephalus (Duffy et al., 2009; Grard et al., 2014; Ledermann et al., 2014; Musso et al., 2018). However, it was also found that ZIKV has other transmission routes such as mother-tochild transmission, sexual transmission and blood transmission (D'Ortenzio et al., 2016; Foy et al., 2011; Lessler et al., 2016; Pingen et al., 2016). ZIKV shows a high tendency and long persistence in human tissues and body fluids (Barzon et al., 2016; Carteaux et al., 2016; Furtado et al., 2016; Joguet et al., 2017; Miner and Diamond, 2017; Tabata et al., 2016). In addition to infecting the eyes, brain, testes, and placenta, the virus can be also detected in tears, urine, saliva, and even semen. Importantly, current studies have reported that ZIKV RNA can persist in the patient's semen for up to six months after the onset of infection (Mead et al., 2018).

Phylogenetic studies have shown that ZIKV has evolved into two lineages, African and Asian, and the ZIKV strains that caused the outbreaks in the Americas belong to the Asian lineage (Musso and Gubler, 2016; Wang et al., 2016). Although the ZIKV epidemics have currently subsided, the re-emerging ZIKV shows a stronger infectious and pathogenic activity than its precursor originating from Africa. Therefore, understanding the molecular mechanisms of ZIKV pathogenicity and biological functions of various viral proteins will help us to treat and prevent ZIKV infection, and also provide references for coping with other emerging and recurrent viruses. This article gives a comprehensive description of the existing functional research of each viral protein of ZIKV and their interactions with host factors, and discusses the possible causes for the infectivity and pathogenicity of ZIKV.

\section{Capsid protein}

The capsid (C) protein is the viral structural protein which, combined with the viral genomic RNA, forms the nucleocapsid (NC) of the ZIKV particles (Shang et al., 2018). The
$\mathrm{NC}$ is enveloped by a lipid membrane containing prM and $\mathrm{E}$ proteins to produce the immature virus particles in the endoplasmic reticulum (ER). Structural studies have shown that the overall structure of the ZIKV C protein is similar to the structure of DENV and WNV C proteins (Shang et al., 2018, Tan et al., 2020). The N-terminus of the C protein contains a highly positively charged loop, followed by five $\alpha$ helices (helix $\alpha 1-\alpha 5$ ). The viral protease NS2B/3 cleaves at the junction of $C$ protein helices $\alpha 4-\alpha 5$ and thus releases the mature $\mathrm{C}$ protein, leaving helix $\alpha 5$ embedded in the ER membrane. The hydrophobic helix $\alpha 1$ helps $\mathrm{C}$ protein dimer production and interacts with the viral lipid membrane (Shang et al., 2018; Tan et al., 2020). The structural analysis of $\mathrm{C}$ protein shows that differences in the sequence of helix $\alpha 1$ have an impact on the properties and the degree of exposure of the hydrophobic cleft, suggesting that helix $\alpha 1$ is a hot spot for evolutionary adaptation of flaviviruses (Morando et al., 2019). Helix $\alpha 4$, with a high positive charge interface, binds to nucleotides, and helix $\alpha 5$ is used as a signal peptide after the cleavage of $\mathrm{C}$ protein to guide prM to enter the ER lumen (Tan et al., 2020). Recent studies revealed that $C$ protein plays a central role in the assembly of ZIKV. The study of Morando et al. suggests that the dynamics of $\mathrm{C}$ protein structural elements responds to a structure-driven regulation of interaction with endocellular hydrophobic interfaces, which are required for $\mathrm{NC}$ assembly (Morando et al., 2019). The study of Tan et al. describes a subnanometer resolution structure of $\mathrm{C}$ proteins within virus particles. It illuminates the quaternary structure of the $\mathrm{C}$ protein and also shows the presence of helix $\alpha 5$ on $\mathrm{C}$ protein (previously thought to be removed by protease hydrolysis). Moreover, helix $\alpha 5$ was verified to be able to induce $\mathrm{C}$ protein dimers which subsequently form larger oligomers during the assembly process. Since E and prM alone cannot produce regular flavivirus icosahedral particles, C protein was suggested to serve as an important mediator between $\mathrm{E} /$ prM and viral genome to ensure the correct assembly of virus particles (Tan et al., 2020). Shang et al. found that in addition to binding to ssRNA, C protein also shows high affinity to dsRNA, dsDNA and ssDNA, indicating that $\mathrm{C}$ protein may participate in nucleotide-related host reactions (Shang et al., 2018).

In addition, some studies have shown that $\mathrm{C}$ protein is involved in post-transcriptional regulations in host cells and the virus-related neurological diseases. Hou et al. found that ZIKV C protein can form a stable complex with Ras-GAP SH3 domain-binding protein 1 (G3BP1) and Caprin-1 to block the formation of stress granules (SGs) in the host cell, thus removing SGs-mediated restriction on the translation of the viral genome (Hou et al., 2017). The disruption of the nonsense-mediated mRNA decay (NMD) pathway is a known cause of microcephaly and other neurological disorders (Fontaine et al., 2018). Fontaine et al. revealed that in 
human neural progenitor cells (hNPCs), ZIKV C protein can bind to the main regulator up-frameshift protein 1 (UPF1) in the NMD pathway and induces its proteasome-dependent degradation, thereby inhibiting the antiviral effect of the NMD pathway and disrupting the cellular process of quality control, eventually leading to neuropathy in the developing brain (Fontaine et al., 2018). No significant difference between viral strains of Asian and African lineages was shown for the interaction between $\mathrm{C}$ protein and the NMD pathway (Fontaine et al., 2018). Recently, a study also reported that $\mathrm{C}$ protein specifically binds and inhibits Dicer to limit host miRNA biogenesis in neural stem cells (NSCs), resulting in neurodevelopmental defects and virus immune evasion (Zeng et al., 2020).

\section{Precursor membrane protein}

The precursor membrane (prM) protein is one of the structural proteins on the surface of the envelope of ZIKV particles, and plays an important role in the assembly and maturation of virus particles. The protein interacts with the $\mathrm{E}$ protein in the ER membrane, and then encapsulates the viral nucleocapsid to produce immature virus particles (Hasan et al., 2018; Nambala and Su, 2018). During the transport of immature virus particles to the Golgi apparatus, prM protects the E protein from premature fusion. After entering the Golgi apparatus, most of the prM is cleaved by the host furin protein to produce a 75 -amino acid $\mathrm{M}$ protein and 99-amino acid Pr polypeptide, after which the virus matures and is released (Li et al., 2019).

Recent studies indicate that prM protein may be related to the neurovirulence by ZIKV infection. After studying the effect of prM amino acid substitutions on ZIKV, Yuan et al. found that in most contemporary epidemic strains, a serine to asparagine substitution (S139N) appeared in prM protein (Yuan et al., 2017). This amino acid substitution, among other substitutions along the genome, showed the greatest neurovirulence in newborn mice. Moreover, after reversing the amino acid substitution (N139S), the neurovirulence of the N139S mutant was lowered. In addition, ZIKV infection of human brain precursor cell (hNPC) has also shown, compared to N139S mutant strains or pre-epidemic Asian strains, that $\mathrm{S} 139 \mathrm{~N}$ can enhance ZIKV replication and cell lethality in hNPC (Yuan et al., 2017). Subsequently, Li et al. showed that prM and Pr peptides can directly induce apoptosis of human brain glial cells SNB-19, but the S139N reverse mutation in the $\operatorname{Pr}$ region did not change its cytotoxicity to SNB-19 (Li et al., 2019). Therefore, these findings indicate that prM may constitute a component of ZIKV cytotoxicity, and prM S139N in the ZIKV epidemic strain is critical to cause nerve-associated diseases by ZIKV infection, possibly by affecting the interaction profile with $\mathrm{E}$ protein and the structural stability of the virions.

\section{Envelope protein}

The envelope (E) protein is the main component of the icosahedral capsule envelope of the ZIKV particles, responsible for the virus attaching to and entry the host cell, as well as for host adaption, immune recognition and virus pathogenesis. It is also the major protein for inducing neutralizing antibodies against virus infection (Dai et al., 2016; Fontes-Garfias et al., 2017; Giraldo et al., 2020; Sapparapu et al., 2016; Shan et al., 2020; Stettler et al., 2016). It has a total length of 505 amino acids, similar to other flavivirus $\mathrm{E}$ proteins, and consists of four domains. The $\beta$-barrel-shaped domain I (DI) connects DII and DIII as the structural center; finger-like domain II (DII) contains the E protein dimerization interface and a fusion loop that interacts with the endoplasmic membrane; the C-terminal immunoglobulin-like domain III (DIII) contains the receptor binding site, which is mediated by the $\mathrm{E}$ protein and plays a role in membrane fusion. The fourth domain is comprised of $\alpha$-helix stem region and an $\alpha$-helix transmembrane region anchored to the ER membrane (Dai et al., 2016; Kostyuchenko et al., 2016). Many groups have identified DII and DIII in the E protein as critical for a potent neutralizing response, designating them as the important regions for developing an effective and safe ZIKV subunit vaccine (Hasan et al., 2017; Sapparapu et al., 2016; Stettler et al., 2016; Tai et al., 2018).

Kostyuchenko and his colleagues have shown that ZIKV has higher thermal stability than DENV. When the virus is incubated at $40^{\circ} \mathrm{C}$, unlike DENV, ZIKV virus particles can still maintain their structural stability, which is most likely due to the tight packing of ZIKV E protein dimers and complex interactions that give the virus a more dense and stable structure (Kostyuchenko et al., 2016). This may explain why ZIKV can withstand harsh conditions and remain in different body fluids such as tears, urine, saliva, and semen. Studies in vitro showed that different cell types are permissive to ZIKV infection (Aagaard et al., 2017; Chen et al., 2016; Hamel et al., 2015; Sheng et al., 2017; Tang et al., 2016). DC-SIGN, HSP70, TIM-1 and the TAM receptors (TYRO3, AXL and MER) have been shown to be beneficial for the entry of ZIKV and other flaviviruses into host cells and are suggested to be the possible candidate receptors/coreceptors (Hamel et al., 2015; Hastings et al., 2017; Laureti et al., 2018; Meertens et al., 2017; Pujhari et al., 2019; Tabata et al., 2016). However, these studies have not shown direct interactions between $\mathrm{E}$ protein and the mentioned host factors, thus the identification of the host receptor(s) still needs further research. Notably, recent research shows that E protein is ubiquitinated by the tripartite motif 7 (TRIM7) through Lys63 (K63)-linked polyubiquitination, which is a 
determinant for ZIKV entry, tissue tropism and pathogenesis (Giraldo et al., 2020).

The E protein N154 glycosylation is required for ZIKV infection of $A$. aegypti and critical for virus virulence in A129 mice. Except for some early African strains, E proteins of the most ZIKV strains contain a glycosylation site at amino acid N154 (Fontes-Garfias et al., 2017). The mutation of this site (N154Q) weakened ZIKV infectivity in A129 mice and $A$. aegypti, but this mutation shows no significant effect on the neurovirulence of ZIKV (Fontes-Garfias et al., 2017). However, removal of the N154 glycosylation in $\mathrm{E}$ protein had little impact on viral infection in mammalian cell but increased viral fitness in A. aegypti (C6/36) cells (FontesGarfias et al., 2017; Gong et al., 2018). These discrepancies observed in vivo and in vitro may be due to a network of cytokines and a complex immune system which lacks in cell lines. Another study suggests that the E protein glycosylation may contribute to ZIKV pathogenesis via enhancing infection of lectin-expressing leukocytes (Carbaugh et al., 2019). Recently, Shan et al. revealed that ZIKV acquired an evolutionary mutation in the E protein (V473M), before its 2013 transmit to the Americas, which is a critical determinant for enhanced neurovirulence, mother-to-child transmission, and viremia to facilitate urban transmission (Shan et al., 2020). This study explains, in a large part, the reason of ZIKV reemergence and enhanced pathogenicity. In addition, Zhou et al. found that ZIKV E protein specifically interacts with major facilitator superfamily domain-containing protein 2 (Mfsd2a) in human brain microvascular endothelial cells (hBMECs), promotes the ubiquitination degradation of Mfsd2a, damages Mfsd2a-mediated intake of docosahexaene acid (DHA), eventually leading to impaired brain development (Zhou et al., 2019).

\section{NS1}

Nonstructural protein 1 (NS1) is a multifunctional nonstructural protein of ZIKV, with a total length of 352 amino acids (Xu et al., 2016). NS1 can exist in two forms, as a homodimer and as a hexamer. The dimeric NS1 is mainly located in the ER lumen of the cell, and is involved in regulating the host cell immune responses and assisting virus replication (Song et al., 2016; Xu et al., 2016). Hexamer NS1 protein is released to the outside of the cell, thereby triggering the host immune responses and inducing hyperpermeability in tissues (Puerta-Guardo et al., 2019). Structural analysis of NS1 by Xu et al. showed that the overall structure of ZIKV NS1 is very similar to that of DENV and WNV NS1, of which the monomer structure has three domains: $\beta$-hairpin domain, wing domain and $\beta$-ladder domain (Xu et al., 2016).

The functional studies on flavivirus NS1 have revealed that NS1 plays an important role in virus replication. Recently, $\mathrm{Ci}$ et al. found that ZIKV NS1 can induce the remodeling of the ER membrane to form a replication compartment for ZIKV (Ci et al., 2020). In addition, NS1 was found to suppress the immune response of host cells through two ways: (1) NS1 directly binds TANK binding kinase 1 (TBK1) and inhibits phosphorylation of TBK1, thus blocking the interferon (IFN) signaling; (2) NS1 recruits USP8 to inhibit the degradation of caspase-1 and then activates NLRP3 inflammatory bodies, thereby reducing the production of IFN (Wu et al., 2017; Xia et al., 2018; Zheng et al., 2018).

As the only protein among ZIKV viral proteins to have an exocrine form among ZIKV viral proteins, there have been relevant studies referring that NS1 plays an important role in virus invasion of several immune-privileged sites. PuertaGuardo et al. found that ZIKV NS1 can specifically induce increased permeability of human umbilical vein endothelial cells in vitro and induce brain endothelial vascular leakage in mice, and NS1s from both African and American strains behaved similarly in that regard (Puerta-Guardo et al., 2019). In addition, Puerta-Guardo et al. also reported that NS1 modulates the glycosaminoglycans (GAGs) on trophoblasts and chorionic villi, causing the placental dysfunction and resulting in increased permeability of human placentas (Puerta-Guardo et al., 2020). Recently, Hui et al. revealed that ZIKV NS1 can disrupt the integrity of the blood-testis barrier (BTB) by interacting with matrix metalloproteinase 9 (MMP9) and facilitated K63-linked polyubiquitination of MMP9, which leads to the degradation of the essential proteins involved in the maintenance of the BTB (Hui et al., 2020). Therefore, apart from promoting viral replication and immune escape, NS1 can promote virus transmission from blood to tissues.

The study by Liu et al. indicated the NS1 A188V can significantly affect the infectivity of the virus among mosquitoes. In their initial research, they found that NS1 facilitates flavivirus acquisition by mosquitoes from an infected mammalian host and subsequently enhances viral prevalence in mosquitoes (Liu et al., 2016). Asian ZIKV isolates from before 2012, with an alanine at residue 188 of NS1, had weak infectivity and low prevalence in mosquitoes and caused only sporadic outbreaks (Liu et al., 2017). In contrast, in ZIKV strains responsible for the epidemic in the Americas, the spontaneous substitution of A188V in its NS1 protein, results in increased NS1 antigenaemia and enhancement of ZIKV infectivity and prevalence in mosquitoes, potentially leading to facilitated transmission (Liu et al., 2017). Amino acid 188 of NS1 is the key point to affect virus transmission ability in mosquitoes, however, it is not a determinant of the viral prevalence among the population, and other unknown factors might be involved (Liu et al., 2017). Subsequently, $\mathrm{Xia}$ et al. further investigated this mutation, and found that 
A188V of NS1 can enhance the binding of NS1 to TBK1, thereby reducing the effect of the IFN response (Xia et al., 2018). Furthermore, the V188A reverse mutation in the South American epidemic strain indicated that this mutation had no effect on the neurovirulence, one of the pathological characteristics of ZIKV-induced diseases during the epidemic (Xia et al., 2018). Thus the mutation of NS1 at position 188 is an important but not the only factor for the transformation of virus infectivity and pathogenicity, and this transformation is more likely to be determined by multiple substitutions along the viral genome and other unknown factors.

\section{NS2A}

NS2A is an ER-resident, membrane-bound protein with a total length of 226 amino acids and a calculated molecular weight of approximately $22 \mathrm{kD}$ (Zhang et al., 2019a; Zhang et al., 2019b). Unfortunately, the protein structure of a flavivirus NS2A has not yet been successfully resolved. But some studies have revealed the topological structure of Dengue virus (DENV) and ZIKV NS2A, which greatly promoted our understanding of how the natural state of NS2A exerts its biological functions (Xie et al., 2013; Zhang et al., 2019b). The biochemical structure study of ZIKV NS2A by Zhang et al. showed that the N-terminal region of NS2A, amino acid residues 1-56, is located on the ER lumen side and contains two membrane-associated segments. Residues 74-97 comprise a transmembrane segment that spans the ER membrane, and residues 103-226, the C-terminal region of NS2A, is located on the cytoplasmic side and contains four membrane-associated segments (Zhang et al., 2019b). In addition, Zhang et al. showed that NS2A is functionally involved in the ZIKV RNA replication and the assembly of viral particles. They also identified the key residues of NS2A that affect viral replication or assembly (Zhang et al., 2019b). The single mutation of NS2A (A175V) described by Márquez-Jurado and her colleagues using reverse genetics can affect viral RNA synthesis and the generation of viral particles, which also confirmed a role of NS2A in virus replication and assembly (Márquez-Jurado et al., 2018). Later, Zhang et al. further studied the molecular mechanism of ZIKV NS2A involved in virus assembly. They found that NS2A recruits viral NS2B/NS3 protease and the structural C-prM-E polyprotein to the virion assembly site. Once the C-prM-E polyprotein has been processed, NS2A binds the 3'-UTR of the progeny viral RNA via its cytoplasmic loop, thus presenting viral RNA to the structural proteins to enable virion assembly (Zhang et al., 2019a).

In addition to playing a direct role in the life cycle of ZIKV, recent studies indicate that NS2A is also involved in regulating the host innate immune response and has causal re- lations with neurological diseases. Xia et al. have shown that NS2A can inhibit the IFN response by participating in the inhibition of TBK1 phosphorylation (Xia et al., 2018); Nguyen et al. have revealed that NS2A can directly inhibit RIG-I and IRF3 to antagonize the RIG-I/MDA5 pathwaymediated production of interferon- $\beta$ (IFN- $\beta$ ) (Ngueyen et al., 2019). Yoon et al. found that NS2A can directly mediate the degradation of adhesion junction proteins, thereby destroying the neurogenesis of mammalian cortex, and similar phenomena were also observed in West Nile virus (WNV) and Japanese encephalitis virus (JEV) infections (Yoon et al., 2017). This phenomenon indicates that NS2A is one of the important causal factors for ZIKV-induced neurovirulence. Of note, NS2A is relatively highly conserved, showing nearly $99.9 \%$ identity at the protein level among different ZIKV strains (Yoon et al., 2017). This indicates that Zika virus may have neurovirulence from the beginning, and NS2A is not the only factor in the pathogenic transformation of Zika virus.

\section{NS2B/3}

NS2B is also an ER-resident, transmembrane protein (Xing et al., 2020). From the existing research, NS2B mainly acts as a cofactor of NS3. NS2B binds and stabilizes the structure of NS3, providing an ER anchor point for NS3 and activating the NS3 active region to exert protease activity ( $\mathrm{Li}$ et al., 2018; Phoo et al., 2016; Xing et al., 2020). Viral polyprotein precursors are processed into mature proteins (three structural proteins and seven non-structural proteins) by host proteases and NS2B/3 proteases (Phoo et al., 2016; Xing et al., 2020). However, there is not much data supporting that NS2B alone could exert biological functions. The study of Xia et al. has mentioned that ZIKV NS2B can inhibit the phosphorylation of TBK1 to suppress IFN- $\beta$ production (Xia et al., 2018).

NS3 is the viral protease and helicase of ZIKV. After expression, it is mainly located in the mitochondria (Xing et al., 2020). NS3 is a common multifunctional viral protein among the Flavivirus members, with $\mathrm{N}$-terminal serine protease domain and C-terminal nucleoside triphosphatase (NTPase) and RNA helicase domains (Lei et al., 2016; Phoo et al., 2016). The protease region of NS3 is mainly used to process the polyprotein precursor produced after the translation of viral genome. However, Xing et al. found that NS3 cannot fully exert its protease activity without binding to NS2B (Xing et al., 2020). The NS3 nucleoside triphosphatase hydrolyzes ATP during viral genome replication to provide energy for dissociating the double-stranded RNA into singlestranded RNA via the NS3 helicase region (Xu et al., 2019). In the study of NS3 helicase activity, Xu et al. found that the interaction between NS5 and NS3 can further activate its 
helicase activity, and blocking this process leads to defects in virus replication (Xu et al., 2019). As NS3 does not have a membrane-bound structure itself, it needs the assistance of NS2B to play its biological function on the ER (Xing et al., 2020).

NS3 plays a key role in the virus life cycle, and its function is generally more conservative in flaviviruses. So far the researches around NS3 or NS2B/3 have mainly focused on the related inhibitors and enzyme activities (Chan et al., 2017; Lei et al., 2016; Li et al., 2018; Xu et al., 2019), only a few studies have reported that NS2B/3 also play a role in viral pathogenicity and host immune regulation. Li et al. first reported that $\mathrm{NS} 2 \mathrm{~B} / 3$ is involved in the neurovirulence caused by ZIKV (Li et al., 2019). They found that NS2B/3 protease cleaves Septin-2 (SEPT2) in human brain neural precursor cells (hNPCs), resulting in a decrease of protein level of SEPT2 to inhibit hNPC cytokinesis, and eventually triggers genotoxic stress and cell death in hNPCs. Sequence alignments associated with comparison of protease activities against Septin-2 of ZIKV NS2B/3 of different strains showed that although the Asian strain had four amino acid changes compared with the African strain, the efficiency of cleaving Septin-2 was not much different between those ZIKV strains (Li et al., 2019). In the same year, Hou and his colleagues reported that NS3 is also involved in inhibiting the formation of SGs to interfere with host translation and ribonucleoprotein complex transport (Hou et al., 2017). In addition, NS2B/ 3 also plays a role in the down-regulation of host immune responses by inhibiting the induction of IFN and downstream IFN-stimulated genes (Wu et al., 2017). Besides, NS2B/3 can suppress activation of JAK-STAT signaling by targeting Jak1 for degradation and attenuate RLR-induced apoptosis to ensure the complement of viral cell life cycle (Wu et al., 2017). Furthermore, NS2B/3 also mediates the cleavage of R78 and G79 in the cytoplasmic loop of human stimulator of IFN genes (hSTING), suppressing the cGAS/STING pathway to blunt cell-intrinsic antiviral defenses (Ding et al., 2018).

\section{NS4A/4B}

NS4A and NS4B are another two membrane-associated nonstructural proteins encoded by ZIKV. Since there have been no reports on either ZIKV or other flavivirus NS4A and NS4B structural studies to date, it is difficult to have a clear picture of how these proteins are structurally organized. In other flavivirus-related studies, NS4B have been reported to be involved in viral replication (Evans and Seeger, 2007), but what the role NS4B plays in ZIKV replication remains unknown. Currently, related functional studies have shown that NS4A/4B is involved in regulating host immune response and triggering nerve-associated diseases.
$\mathrm{Wu}$ et al. found that NS4B can combine with TBK1 to inhibit the production of type I interferon, which is also reported by Xia et al. (Wu et al., 2017; Xia et al., 2018). Furthermore, NS4A blocks interferon regulatory factor 3 (IRF3) phosphorylation, thus preventing the production of type I IFN induced by the melanoma differentiation associated gene 5 (MDA5)/retinoic acid-inducible gene I (RIG-I) signaling pathway (Hu et al., 2019; Ma et al., 2018; Ngueyen et al., 2019; Xia et al., 2018). The research of Ma et al. showed that NS4A combines with MAVS to hinder the interaction between mitochondrial antiviral signaling protein (MAVS) and RIG-I and thus reduces the induction of type I IFN, which is also supported by Hu et al. (Hu et al., 2019; Ma et al., 2018). Interestingly, type III IFN protects human placental barrier cells from ZIKV infection, and this type of IFN production is also regulated by the MAVS signaling ( Hu et al., 2019; Lazear et al., 2015). Therefore, it may explain from another perspective how ZIKV crosses the placenta during pregnancy to infect the developing fetus. In addition, NS4A is also involved in regulation of host cell metabolism by inhibiting the formation of SGs and triggering the overall translational block of host cells to assist in virus replication (Hou et al., 2017).

In 2016, Liang and his colleagues reported that NS4A/4B plays a major role in the pathogenesis of neonatal microcephaly (Liang et al., 2016). They found that NS4A/4B can interfere with Akt (also known as protein kinase B)-mammalian target of rapamycin (mTOR) signal transduction in human fetal neural stem cells (fNSCs) by inhibiting Akt phosphorylation. The Akt-mTOR signaling pathway regulates the neurogenesis and inhibits cell autophagy of fNSCs (Liang et al., 2016). Therefore, when ZIKV NS4A and NS4B inhibit the Akt-mTOR signaling pathway, this in turn impedes the neurogenesis of fNSCs and increases autophagy. Moreover, comparison experiments of representative ZIKV strains of Asian and African lineage (MR766, IbH30656, and $\mathrm{H} / \mathrm{PF} / 2013$ ) showed similar levels of neurogenesis inhibition in fNSCs, consistent with the high sequence identity of NS4A and NS4B among those strains (Liang et al., 2016). It is therefore likely that additional factors, such as key amino acids in the sequence other than NS4A/4B, are associated with pathogenesis of the epidemic ZIKV strains. The Ankryin repeat and LEM domain containing 2 (ANKLE2)-Ball (VRK1) pathway is required for proper localization of asymmetric proteins and spindle alignment during neuroblast (NB) cells division, and mutations in ANKLE2 cause autosomal recessive microcephaly (Link et al., 2019; Yamamoto et al., 2014). Exogenous expression of NS4A in NB cells mimics microcephaly phenotypes seen in ANKLE2 mutants. The underlying mechanism might be its specific interaction with ANKLE2 and blockage of the downstream signal transduction, leading to defects in brain development (Link et al., 2019; Shah et al., 2018). 


\section{NS5}

NS5 is the largest non-structural protein encoded by ZIKV, with a full length of 903 amino acids, consisting of two major functional domains: the $\mathrm{N}$-terminal methyltransferase (MTase) domain and the C-terminal RNA-dependent RNA polymerase (RdRP) domain (Wang et al., 2017; Zhao et al., 2017). The main biological function of NS5 is being responsible for viral RNA synthesis and capping. Structural analysis shows that the overall structure of ZIKV NS5 is very similar to JEV NS5 (Zhao et al., 2017). The ZIKV NS5 protein from African and Brazilian strains has a similar RNA synthesis capacity although there are 36 amino acid residue differences on their protein surface (Zhao et al., 2017).

Apart from its own enzymatic functions, research in recent years has shown that NS5 is also involved in the immune regulation of host cells. Grant et al. revealed that ZIKV NS5 can bind and induce ubiquitination-mediated degradation of

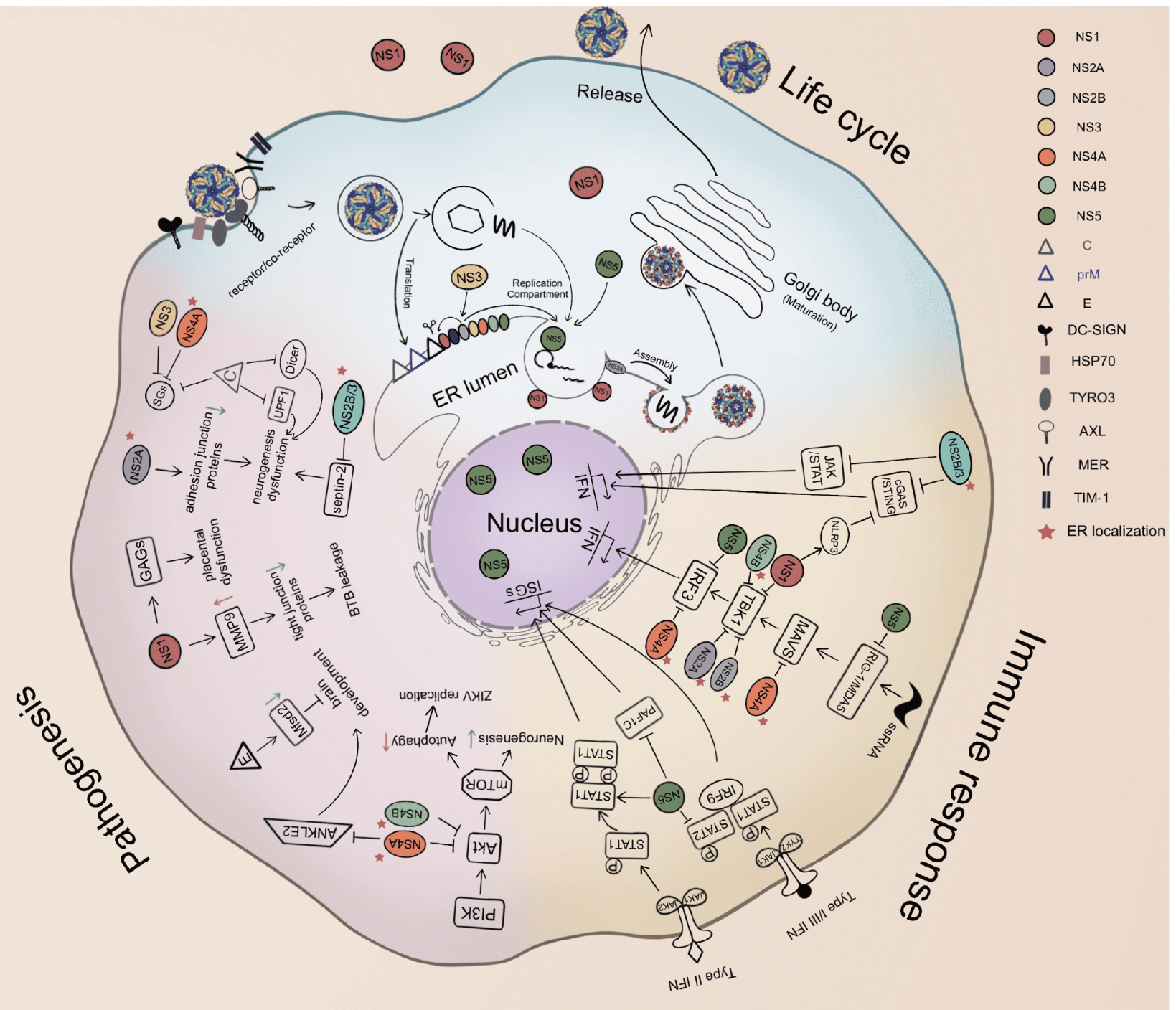

Figure 1 Schematic representation of network interactions of ZIKV proteins and host factors. The functions of ZIKV nonstructural proteins in restricting host antiviral immunity: ZIKV NS1, NS2A, NS2B, NS4A, NS4B and NS5 negatively regulate RIG-I-like receptor-induced IFN- $\beta$ production; NS1 activates NLRP3 inflammatory bodies by inhibiting the degradation of caspase-1; NS2B/3 blocks the JAK-STAT signal transduction and suppresses the cGAS/STING pathway; NS5 induces ubiquitination-mediated degradation of human STAT2, and blocks STAT1 phosphorylation and enhances the type II interferon response by promoting the formation of STAT1-STAT1 protein complexes; NS5 suppresses ISGs by inhibiting PAF1C recruitment. The functions of ZIKV proteins in viral pathogenicity: C protein degrades UPF1 and inhibits Dicer resulting in neurogenesis dysfunction; NS1 disrupts the integrity of the BTB by upregulating the protein level of MMP9 and increases the permeability in developing human placentas by disrupting GAGs; E protein induces the degradation of Mfsd2a to impede brain development; ZIKV NS2A inhibits neurogenesis by degrading adhesion junction proteins; NS2B/3 protease causes genotoxic stress and cell death by cleaving Septin-2 leading to neurogenesis dysfunction; NS4A inhibits ANKLE2 to cause microcephaly; NS4A and NS4B blocks the neurogenesis by deregulating Akt-mTOR signaling; C protein, NS3 and NS4A negatively regulate the formation of SGs to interfere with host translation and the ribonucleoprotein complex. The functions of ZIKV nonstructural proteins in the viral life cycle: NS1 induces the ER to form the replication compartment; NS2B/3 cleaves the viral polyprotein and dissociates the double-stranded RNA; NS5 is responsible for viral RNA synthesis and capping; NS2A recruits the complexes for virion assembly to the ER (Mature ZIKV particle: PDB 5IZ7. Immature ZIKV particle: PDB 6LNU). 
human (but not murine) STAT2 to antagonize the type I IFN response (Grant et al., 2016). Therefore, ZIKV is also likely to suppress the type III IFN signaling by targeting STAT2, enabling ZIKV to infect the fetus through the placenta. Kumar et al. also confirmed that ZIKV infection inhibited type I and type III IFN signal transduction, and further showed that NS5 mediates STAT2 degradation through its MTase domain interacting with STAT2. In the study of Bowen et al., it was shown that ZIKV can antagonize IFNmediated phosphorylation of STAT1 and STAT2 to inhibit IFN responses. Further research by Chaudhary et al. demonstrated that NS5, in addition to inducing ubiquitination degradation of STAT2, also promotes the formation of STAT1-STAT1 protein complexes, thereby enhancing the STAT1-mediated type II IFN response (Chaudhary et al., 2017). Hertzog and his colleagues also found that NS5 can block STAT1 phosphorylation during viral infection (Hertzog et al., 2018), while Shah et al. found that the NS5 protein inhibits the expression of IFN-stimulated genes (ISGs) by impeding the recruitment of the PAF1 complex (PAF1C) (Shah et al., 2018). Moreover, NS5 was also reported to repress K63-linked polyubiquitination of RIG-I, attenuate the phosphorylation and nuclear translocation of IRF3, and inhibit the production of IFN- $\beta$ (Li et al., 2020; Xia et al., 2018).

ZIKV NS5 was revealed to be localized in the nucleus after expression (Shah et al., 2018; Zhang et al., 2019a), which is also observed with DENV NS5 that translocates, after release from the polypeptide, to the nucleus and then hampers the splicing of host mRNA (De Maio et al., 2016). Interestingly, according to a recent study, the ZIKV NS5 nuclear accumulation protects NS5 from cytoplasmic degradation, enabling NS5 to perform its biological functions in the virus life cycle (Ji and Luo, 2020).

\section{Perspective}

The re-emerging of ZIKV and its link with various clinical diseases led to a rapid research response in the world, which has provided some answers to why this virus transitioned from mild to notorious. To date, the studies of ZIKV proteins have partially answered questions about viral persistence, evolution, virulence, tissues tropism, immune evasion, and virus transmission dynamics, and the mechanism of ZIKV causing neurodevelopmental disease (Figure 1). However, there is still too limited knowledge about the ZIKV receptor, the structure of membrane-bound nonstructural proteins, as well as the mechanism of male-reproductive sequelae of virus infection. ZIKV infection has been posing a great threat to public health, yet, there are no available drugs, vaccines or therapeutics against ZIKV infections on the market so far. It is becoming extremely urgent to develop effective anti-
ZIKV drugs for related disease treatment and prevention. Extensive research on the molecular mechanisms of viral proteins and their roles in virus-host interactions will provide clues for the future design of specific drugs disrupting these interactions and reducing the health damage to the host and also shed light on the development of effective vaccines. Finally, the lessons we have learnt from ZIKV may be applicable to other emerging or re-emerging viruses that result in unforeseen human diseases.

Compliance and ethics The author(s) declare that they have no conflict of interest.

Acknowledgements We thank Xinjie Nie at Tianjin Academy of Fine Arts, School of Experimental Art, Tianjin, China for drawing Figure 1.

\section{References}

Aagaard, K.M., Lahon, A., Suter, M.A., Arya, R.P., Seferovic, M.D., Vogt, M.B., Hu, M., Stossi, F., Mancini, M.A., Harris, R.A., et al. (2017). Primary human placental trophoblasts are permissive for Zika virus (ZIKV) replication. Sci Rep 7, 41389.

Barzon, L., Pacenti, M., Berto, A., Sinigaglia, A., Franchin, E., Lavezzo, E., Brugnaro, P., and Palù, G. (2016). Isolation of infectious Zika virus from saliva and prolonged viral RNA shedding in a traveller returning from the Dominican Republic to Italy, January 2016. Eurosurveillance $21,30159$.

Cao-Lormeau, V.M., Roche, C., Teissier, A., Robin, E., Berry, A.L., Mallet, H.P., Sall, A.A., and Musso, D. (2014). Zika virus, French polynesia, South pacific, 2013. Emerg Infect Dis 20, 1084-1086.

Carbaugh, D.L., Baric, R.S., and Lazear, H.M. (2019). Envelope protein glycosylation mediates Zika virus pathogenesis. J Virol 93, e00113-19.

Carteaux, G., Maquart, M., Bedet, A., Contou, D., Brugières, P., Fourati, S., Cleret de Langavant, L., de Broucker, T., Brun-Buisson, C., LeparcGoffart, I., et al. (2016). Zika virus associated with meningoencephalitis. N Engl J Med 374, 1595-1596.

Chan, J.F.W., Chik, K.K.H., Yuan, S., Yip, C.C.Y., Zhu, Z., Tee, K.M., Tsang, J.O.L., Chan, C.C.S., Poon, V.K.M., Lu, G., et al. (2017). Novel antiviral activity and mechanism of bromocriptine as a Zika virus NS2B-NS3 protease inhibitor. Antiviral Res 141, 29-37.

Chaudhary, V., Yuen, K.S., Chan, J.F.W., Chan, C.P., Wang, P.H., Cai, J.P., Zhang, S., Liang, M., Kok, K.H., Chan, C.P., et al. (2017). Selective activation of type II interferon signaling by Zika virus NS5 protein. J Virol 91, e00163-17.

Chen, J.C., Wang, Z., Huang, H., Weitz, S.H., Wang, A., Qiu, X., Baumeister, M.A., and Uzgiris, A. (2016). Infection of human uterine fibroblasts by Zika virus in vitro: implications for viral transmission in women. Int J Infect Dis 51, 139-140.

Ci, Y., Liu, Z.Y., Zhang, N.N., Niu, Y., Yang, Y., Xu, C., Yang, W., Qin, C. F., and Shi, L. (2020). Zika NS1-induced ER remodeling is essential for viral replication. J Cell Biol 219.

D’Ortenzio, E., Matheron, S., Yazdanpanah, Y., de Lamballerie, X., Hubert, B., Piorkowski, G., Maquart, M., Descamps, D., Damond, F., and Leparc-Goffart, I. (2016). Evidence of sexual transmission of Zika virus. N Engl J Med 374, 2195-2198.

Dai, L., Song, J., Lu, X., Deng, Y.Q., Musyoki, A.M., Cheng, H., Zhang, Y., Yuan, Y., Song, H., Haywood, J., et al. (2016). Structures of the Zika virus envelope protein and its complex with a flavivirus broadly protective antibody. Cell Host Microbe 19, 696-704.

De Maio, F.A., Risso, G., Iglesias, N.G., Shah, P., Pozzi, B., Gebhard, L.G., Mammi, P., Mancini, E., Yanovsky, M.J., Andino, R., et al. (2016). The Dengue virus NS5 protein intrudes in the cellular spliceosome and modulates splicing. PLoS Pathog 12, e1005841.

de Oliveira, W.K., de França, G.V.A., Carmo, E.H., Duncan, B.B., de Souza 
Kuchenbecker, R., and Schmidt, M.I. (2017). Infection-related microcephaly after the 2015 and 2016 Zika virus outbreaks in Brazil: a surveillance-based analysis. Lancet 390, 861-870.

Dick, G.W.A., and Haddow, A.J. (1952). Uganda S virus. Trans R Soc Tropical Med Hygiene 46, 600-618.

Dick, G.W.A., Kitchen, S.F., and Haddow, A.J. (1952). Zika Virus (I). Isolations and serological specificity. Trans $\mathrm{R}$ Soc Tropical Med Hygiene 46, 509-520.

Ding, Q., Gaska, J.M., Douam, F., Wei, L., Kim, D., Balev, M., Heller, B., and Ploss, A. (2018). Species-specific disruption of STING-dependent antiviral cellular defenses by the Zika virus NS2B3 protease. Proc Natl Acad Sci USA 115, E6310-E6318.

Duffy, M.R., Chen, T.H., Hancock, W.T., Powers, A.M., Kool, J.L., Lanciotti, R.S., Pretrick, M., Marfel, M., Holzbauer, S., Dubray, C., et al. (2009). Zika virus outbreak on Yap Island, Federated States of Micronesia. N Engl J Med 360, 2536-2543.

Evans, J.D., and Seeger, C. (2007). Differential effects of mutations in NS4B on West Nile virus replication and inhibition of interferon signaling. J Virol 81, 11809-11816.

Fauci, A.S., and Morens, D.M. (2016). Zika Virus in the Americas-Yet another arbovirus threat. N Engl J Med 374, 601-604.

Faye, O., Freire, C.C.M., Iamarino, A., Faye, O., de Oliveira, J.V.C., Diallo, M., Zanotto, P.M.A., and Sall, A.A. (2014). Molecular evolution of Zika virus during its emergence in the 20th century. PLoS Negl Trop Dis 8, e2636.

Fontaine, K.A., Leon, K.E., Khalid, M.M., Tomar, S., Jimenez-Morales, D., Dunlap, M., Kaye, J.A., Shah, P.S., Finkbeiner, S., Krogan, N.J., et al. (2018). The cellular NMD pathway restricts Zika virus infection and is targeted by the viral capsid protein. mBio 9 .

Fontes-Garfias, C.R., Shan, C., Luo, H., Muruato, A.E., Medeiros, D.B.A., Mays, E., Xie, X., Zou, J., Roundy, C.M., Wakamiya, M., et al. (2017). Functional analysis of glycosylation of Zika virus envelope protein. Cell Rep 21, 1180-1190.

Foy, B.D., Kobylinski, K.C., Chilson Foy, J.L., Blitvich, B.J., Travassos da Rosa, A., Haddow, A.D., Lanciotti, R.S., and Tesh, R.B. (2011). Probable non-vector-borne transmission of Zika virus, Colorado, USA Emerg Infect Dis 17, 880-882.

Furtado, J.M., Espósito, D.L., Klein, T.M., Teixeira-Pinto, T., and da Fonseca, B.A. (2016). Uveitis associated with Zika virus infection. N Engl J Med 375, 394-396.

Giraldo, M.I., Xia, H., Aguilera-Aguirre, L., Hage, A., van Tol, S., Shan, C., Xie, X., Sturdevant, G.L., Robertson, S.J., McNally, K.L., et al. (2020). Envelope protein ubiquitination drives entry and pathogenesis of Zika virus. Nature 585, 414-419.

Gong, D., Zhang, T.H., Zhao, D., Du, Y., Chapa, T.J., Shi, Y., Wang, L., Contreras, D., Zeng, G., Shi, P.Y., et al. (2018). High-throughput fitness profiling of Zika virus E protein reveals different roles for glycosylation during infection of mammalian and mosquito cells. iScience 1,97-111.

Grant, A., Ponia, S.S., Tripathi, S., Balasubramaniam, V., Miorin, L., Sourisseau, M., Schwarz, M.C., Sánchez-Seco, M.P., Evans, M.J., Best, S.M., et al. (2016). Zika virus targets human STAT2 to inhibit type I interferon signaling. Cell Host Microbe 19, 882-890.

Grard, G., Caron, M., Mombo, I.M., Nkoghe, D., Mboui Ondo, S., Jiolle, D., Fontenille, D., Paupy, C., Leroy, E.M. (2014). Zika virus in Gabon (Central Africa)-2007: a new threat from Aedes albopictus? PLoS Negl Trop Dis 8, e2681.

Hamel, R., Dejarnac, O., Wichit, S., Ekchariyawat, P., Neyret, A., Luplertlop, N., Perera-Lecoin, M., Surasombatpattana, P., Talignani, L., Thomas, F., et al. (2015). Biology of Zika virus infection in human skin cells. J Virol 89, 8880-8896.

Hasan, S.S., Miller, A., Sapparapu, G., Fernand ez, E., Klose, T., Long, F., Fokine, A., Porta, J.C., Jiang, W., Diamond, M.S., et al. (2017). A human antibody against Zika virus crosslinks the E protein to prevent infection. Nat Commun 8, 14722.

Hasan, S.S., Sevvana, M., Kuhn, R.J., and Rossmann, M.G. (2018). Structural biology of Zika virus and other flaviviruses. Nat Struct Mol Biol 25, 13-20.
Hastings, A.K., Yockey, L.J., Jagger, B.W., Hwang, J., Uraki, R., Gaitsch, H.F., Parnell, L.A., Cao, B., Mysorekar, I.U., Rothlin, C.V., et al. (2017). TAM receptors are not required for Zika virus infection in mice. Cell Rep 19, 558-568.

Hertzog, J., Dias Junior, A.G., Rigby, R.E., Donald, C.L., Mayer, A., Sezgin, E., Song, C., Jin, B., Hublitz, P., Eggeling, C., et al. (2018). Infection with a Brazilian isolate of Zika virus generates RIG-I stimulatory RNA and the viral NS5 protein blocks type I IFN induction and signaling. Eur J Immunol 48, 1120-1136.

Hou, S., Kumar, A., Xu, Z., Airo, A.M., Stryapunina, I., Wong, C.P., Branton, W., Tchesnokov, E., Götte, M., Power, C., et al. (2017). Zika virus hijacks stress granule proteins and modulates the host stress response. J Virol 91, e00474-17.

Hu, Y., Dong, X., He, Z., Wu, Y., Zhang, S., Lin, J., Yang, Y., Chen, J., An, S., Yin, Y., et al. (2019). Zika virus antagonizes interferon response in patients and disrupts RIG-I-MAVS interaction through its CARD-TM domains. Cell Biosci 9, 46.

Hui, L., Nie, Y., Li, S., Guo, M., Yang, W., Huang, R., Chen, J., Liu, Y., Lu, X., Chen, Z., et al. (2020). Matrix metalloproteinase 9 facilitates Zika virus invasion of the testis by modulating the integrity of the bloodtestis barrier. PLoS Pathog 16, e1008509.

Ji, W., and Luo, G. (2020). Zika virus NS5 nuclear accumulation is protective of protein degradation and is required for viral RNA replication. Virology 541, 124-135.

Joguet, G., Mansuy, J.M., Matusali, G., Hamdi, S., Walschaerts, M., Pavili, L., Guyomard, S., Prisant, N., Lamarre, P., Dejucq-Rainsford, N., et al. (2017). Effect of acute Zika virus infection on sperm and virus clearance in body fluids: a prospective observational study. Lancet Infect Dis 17, 1200-1208.

Johansson, M.A., Mier-y-Teran-Romero, L., Reefhuis, J., Gilboa, S.M., and Hills, S.L. (2016). Zika and the risk of microcephaly. N Engl J Med $375,1-4$

Kostyuchenko, V.A., Lim, E.X.Y., Zhang, S., Fibriansah, G., Ng, T.S., Ooi, J.S.G., Shi, J., and Lok, S.M. (2016). Structure of the thermally stable Zika virus. Nature 533, 425-428.

Laureti, M., Narayanan, D., Rodriguez-Andres, J., Fazakerley, J.K., and Kedzierski, L. (2018). Flavivirus receptors: diversity, identity, and cell entry. Front Immunol 9, 2180.

Lazear, H.M., Nice, T.J., and Diamond, M.S. (2015). Interferon- $\lambda$ : immune functions at barrier surfaces and beyond. Immunity $43,15-28$.

Ledermann, J.P., Guillaumot, L., Yug, L., Saweyog, S.C., Tided, M., Machieng, P., Pretrick, M., Marfel, M., Griggs, A., Bel, M., et al. (2014). Aedes hensilli as a potential vector of Chikungunya and Zika viruses. PLoS Negl Trop Dis 8, e3188.

Lei, J., Hansen, G., Nitsche, C., Klein, C.D., Zhang, L., and Hilgenfeld, R. (2016). Crystal structure of Zika virus NS2B-NS3 protease in complex with a boronate inhibitor. Science 353, 503-505.

Lessler, J., Chaisson, L.H., Kucirka, L.M., Bi, Q., Grantz, K., Salje, H., Carcelen, A.C., Ott, C.T., Sheffield, J.S., Ferguson, N.M., et al. (2016). Assessing the global threat from Zika virus. Science 353, aaf8160.

Li, A., Wang, W., Wang, Y., Chen, K., Xiao, F., Hu, D., Hui, L., Liu, W., Feng, Y., Li, G., et al. (2020). NS5 conservative site is required for Zika virus to restrict the RIG-I signaling. Front Immunol 11, 51.

Li, G., Bos, S., Tsetsarkin, K.A., Pletnev, A.G., Desprès, P., Gadea, G., and Zhao, R.Y. (2019). The roles of prM-E proteins in historical and epidemic Zika virus-mediated infection and neurocytotoxicity. Viruses $11,157$.

Li, H., Saucedo-Cuevas, L., Yuan, L., Ross, D., Johansen, A., Sands, D., Stanley, V., Guemez-Gamboa, A., Gregor, A., Evans, T., et al. (2019). Zika virus protease cleavage of host protein septin-2 mediates mitotic defects in neural progenitors. Neuron 101, 1089-1098.e4.

Li, Y., Loh, Y.R., Hung, A.W., and Kang, C.B. (2018). Characterization of molecular interactions between Zika virus protease and peptides derived from the C-terminus of NS2B. Biochem Biophys Res Commun 503, 691-696.

Li, Y., Zhang, Z., Phoo, W.W., Loh, Y.R., Li, R., Yang, H.Y., Jansson, A.E., Hill, J., Keller, T.H., Nacro, K., et al. (2018). Structural insights into the 
inhibition of Zika virus NS2B-NS3 protease by a small-molecule inhibitor. Structure 26, 555-564.e3.

Liang, Q., Luo, Z., Zeng, J., Chen, W., Foo, S.S., Lee, S.A., Ge, J., Wang, S., Goldman, S.A., Zlokovic, B.V., et al. (2016). Zika virus NS4A and NS4B proteins deregulate Akt-mTOR signaling in human fetal neural stem cells to inhibit neurogenesis and induce autophagy. Cell Stem Cell 19, 663-671.

Link, N., Chung, H., Jolly, A., Withers, M., Tepe, B., Arenkiel, B.R., Shah, P.S., Krogan, N.J., Aydin, H., Geckinli, B.B., et al. (2019). Mutations in ANKLE2, a ZIKA virus target, disrupt an asymmetric cell division pathway in Drosophila neuroblasts to cause microcephaly. Dev Cell 51, 713-729.e6.

Liu, J., Liu, Y., Nie, K., Du, S., Qiu, J., Pang, X., Wang, P., and Cheng, G. (2016). Flavivirus NS1 protein in infected host sera enhances viral acquisition by mosquitoes. Nat Microbiol 1, 16087.

Liu, Y., Liu, J., Du, S., Shan, C., Nie, K., Zhang, R., Li, X.F., Zhang, R., Wang, T., Qin, C.F., et al. (2017). Evolutionary enhancement of Zika virus infectivity in Aedes aegypti mosquitoes. Nature 545, 482-486.

Ma, J., Ketkar, H., Geng, T., Lo, E., Wang, L., Xi, J., Sun, Q., Zhu, Z., Cui, Y., Yang, L., et al. (2018). Zika virus non-structural protein 4A blocks the RLR-MAVS signaling. Front Microbiol 9, 1350.

Márquez-Jurado, S., Nogales, A., Ávila-Pérez, G., Iborra, F.J., MartínezSobrido, L., and Almazán, F. (2018). An alanine-to-valine substitution in the residue 175 of Zika virus NS2A protein affects viral RNA synthesis and attenuates the virus in vivo. Viruses 10, 547.

Mead, P.S., Duggal, N.K., Hook, S.A., Delorey, M., Fischer, M., Olzenak McGuire, D., Becksted, H., Max, R.J., Anishchenko, M., Schwartz, A. M., et al. (2018). Zika virus shedding in semen of symptomatic infected men. N Engl J Med 378, 1377-1385.

Meertens, L., Labeau, A., Dejarnac, O., Cipriani, S., Sinigaglia, L., BonnetMadin, L., Le Charpentier, T., Hafirassou, M.L., Zamborlini, A., CaoLormeau, V.M., et al. (2017). Axl mediates ZIKA virus entry in human glial cells and modulates innate immune responses. Cell Rep 18, 324333.

Miner, J.J., and Diamond, M.S. (2017). Zika virus pathogenesis and tissue tropism. Cell Host Microbe 21, 134-142.

Morando, M.A., Barbosa, G.M., Cruz-Oliveira, C., Da Poian, A.T., and Almeida, F.C.L. (2019). Dynamics of Zika virus capsid protein in solution: the properties and exposure of the hydrophobic cleft are controlled by the $\alpha$-helix 1 sequence. Biochemistry acs. biochem.9b00194.

Musso, D., Bossin, H., Mallet, H.P., Besnard, M., Broult, J., Baudouin, L., Levi, J.E., Sabino, E.C., Ghawche, F., Lanteri, M.C., et al. (2018). Zika virus in French Polynesia 2013-14: anatomy of a completed outbreak. Lancet Infect Dis 18, e172-e182.

Musso, D., and Gubler, D.J. (2016). Zika virus. Clin Microbiol Rev 29, 487-524.

Nambala, P., and Su, W.C. (2018). Role of Zika virus prM protein in viral pathogenicity and use in vaccine development. Front Microbiol 9, 1797.

Ngueyen, T.T.N., Kim, S.J., Lee, J.Y., and Myoung, J. (2019). Zika virus proteins NS2A and NS4A are major antagonists that reduce IFN-beta promoter activity induced by the MDA5/RIG-I signaling pathway. J Microbiol Biotech 29, 1665-1674.

Oehler, E., Watrin, L., Larre, P., Leparc-Goffart, I., Lastere, S., Valour, F., Baudouin, L., Mallet, H., Musso, D., and Ghawche, F. (2014). Zika virus infection complicated by Guillain-Barre syndrome-case report, French Polynesia, December 2013. Euro Surveill 19, 4-6.

Phoo, W.W., Li, Y., Zhang, Z., Lee, M.Y., Loh, Y.R., Tan, Y.B., Ng, E.Y., Lescar, J., Kang, C.B., and Luo, D. (2016). Structure of the NS2B-NS3 protease from Zika virus after self-cleavage. Nat Commun 7, 13410.

Pierson, T.C., and Diamond, M.S. (2018). The emergence of Zika virus and its new clinical syndromes. Nature 560, 573-581.

Pingen, M., Bryden, S.R., Pondeville, E., Schnettler, E., Kohl, A., Merits, A., Fazakerley, J.K., Graham, G.J., and McKimmie, C.S. (2016). Host inflammatory response to mosquito bites enhances the severity of arbovirus infection. Immunity 44, 1455-1469.
Puerta-Guardo, H., Glasner, D.R., Espinosa, D.A., Biering, S.B., Patana, M., Ratnasiri, K., Wang, C., Beatty, P.R., and Harris, E. (2019). Flavivirus NS1 triggers tissue-specific vascular endothelial dysfunction reflecting disease tropism. Cell Rep 26, 1598-1613.e8.

Puerta-Guardo, H., Tabata, T., Petitt, M., Dimitrova, M., Glasner, D.R., Pereira, L., and Harris, E. (2020). Zika Virus nonstructural protein 1 disrupts glycosaminoglycans and causes permeability in developing human placentas. J Infect Dis 221, 313-324.

Pujhari, S., Brustolin, M., Macias, V.M., Nissly, R.H., Nomura, M., Kuchipudi, S.V., and Rasgon, J.L. (2019). Heat shock protein 70 (Hsp70) mediates Zika virus entry, replication, and egress from host cells. Emerg Microb Infect 8, 8-16.

Sapparapu, G., Fernandez, E., Kose, N., Fox, J.M., Bombardi, R.G., Zhao, H., Nelson, C.A., Bryan, A.L., Barnes, T., Davidson, E., et al. (2016). Neutralizing human antibodies prevent Zika virus replication and fetal disease in mice. Nature 540, 443-447.

Shah, P.S., Link, N., Jang, G.M., Sharp, P.P., Zhu, T., Swaney, D.L., Johnson, J.R., Von Dollen, J., Ramage, H.R., Satkamp, L., et al. (2018). Comparative flavivirus-host protein interaction mapping reveals mechanisms of Dengue and Zika virus pathogenesis. Cell 175, 19311945.e18.

Shan, C., Xia, H., Haller, S.L., Azar, S.R., Liu, Y., Liu, J., Muruato, A.E., Chen, R., Rossi, S.L., Wakamiya, M., et al. (2020). A Zika virus envelope mutation preceding the 2015 epidemic enhances virulence and fitness for transmission. Proc Natl Acad Sci USA 117, 20190-20197.

Shang, Z., Song, H., Shi, Y., Qi, J., and Gao, G.F. (2018). Crystal structure of the capsid protein from Zika virus. J Mol Biol 430, 948-962.

Sheng, Z.Y., Gao, N., Wang, Z.Y., Cui, X.Y., Zhou, D.S., Fan, D.Y., Chen, H., Wang, P.G., and An, J. (2017). Sertoli cells are susceptible to ZIKV infection in mouse testis. Front Cell Infect Microbiol 7, 272.

Shi, Y., and Gao, G.F. (2017). Structural biology of the Zika virus. Trends Biochem Sci 42, 443-456.

Song, H., Qi, J., Haywood, J., Shi, Y., and Gao, G.F. (2016). Zika virus NS1 structure reveals diversity of electrostatic surfaces among flaviviruses. Nat Struct Mol Biol 23, 456-458.

Stettler, K., Beltramello, M., Espinosa, D.A., Graham, V., Cassotta, A., Bianchi, S., Vanzetta, F., Minola, A., Jaconi, S., Mele, F., et al. (2016). Specificity, cross-reactivity, and function of antibodies elicited by Zika virus infection. Science 353, 823-826.

Tabata, T., Petitt, M., Puerta-Guardo, H., Michlmayr, D., Wang, C., FangHoover, J., Harris, E., and Pereira, L. (2016). Zika virus targets different primary human placental cells, suggesting two routes for vertical transmission. Cell Host Microbe 20, 155-166.

Tai, W., He, L., Wang, Y., Sun, S., Zhao, G., Luo, C., Li, P., Zhao, H., Fremont, D.H., Li, F., et al. (2018). Critical neutralizing fragment of Zika virus EDIII elicits cross-neutralization and protection against divergent Zika viruses. Emerg Microb Infect 7, 1-8.

Tan, T.Y., Fibriansah, G., Kostyuchenko, V.A., Ng, T.S., Lim, X.X., Zhang, S., Lim, X.N., Wang, J., Shi, J., Morais, M.C., et al. (2020). Capsid protein structure in Zika virus reveals the flavivirus assembly process. Nat Commun 11, 895.

Tang, H., Hammack, C., Ogden, S.C., Wen, Z., Qian, X., Li, Y., Yao, B., Shin, J., Zhang, F., Lee, E.M., et al. (2016). Zika virus infects human cortical neural progenitors and attenuates their growth. Cell Stem Cell $18,587-590$.

Wang, B., Tan, X.F., Thurmond, S., Zhang, Z.M., Lin, A., Hai, R., and Song, J. (2017). The structure of Zika virus NS5 reveals a conserved domain conformation. Nat Commun 8, 14763.

Wang, L., Valderramos, S.G., Wu, A., Ouyang, S., Li, C., Brasil, P., Bonaldo, M., Coates, T., Nielsen-Saines, K., Jiang, T., et al. (2016). From mosquitos to humans: genetic evolution of Zika virus. Cell Host Microbe 19, 561-565.

Wu, Y., Liu, Q., Zhou, J., Xie, W., Chen, C., Wang, Z., Yang, H., and Cui, J. (2017). Zika virus evades interferon-mediated antiviral response through the co-operation of multiple nonstructural proteins in vitro. Cell Discov 3, 17006.

Xia, H., Luo, H., Shan, C., Muruato, A.E., Nunes, B.T.D., Medeiros, D.B. 
A., Zou, J., Xie, X., Giraldo, M.I., Vasconcelos, P.F.C., et al. (2018). An evolutionary NS1 mutation enhances Zika virus evasion of host interferon induction. Nat Commun 9, 414.

Xie, X., Gayen, S., Kang, C.B., Yuan, Z., and Shi, P.Y. (2013). Membrane topology and function of dengue virus NS2A protein. J Virol 87, 46094622.

Xing, H., Xu, S., Jia, F., Yang, Y., Xu, C., Qin, C., and Shi, L. (2020). Zika NS2B is a crucial factor recruiting NS3 to the ER and activating its protease activity. Virus Res 275, 197793.

Xu, S., Ci, Y., Wang, L., Yang, Y., Zhang, L., Xu, C., Qin, C., and Shi, L. (2019). Zika virus NS3 is a canonical RNA helicase stimulated by NS5 RNA polymerase. Nucleic Acids Res 47, 8693-8707.

Xu, X., Song, H., Qi, J., Liu, Y., Wang, H., Su, C., Shi, Y., and Gao, G.F. (2016). Contribution of intertwined loop to membrane association revealed by Zika virus full-length ${ }^{\mathrm{NS}} 1$ structure. EMBO J 35, 21702178.

Yamamoto, S., Jaiswal, M., Charng, W.L., Gambin, T., Karaca, E., Mirzaa, G., Wiszniewski, W., Sandoval, H., Haelterman, N.A., Xiong, B., et al. (2014). A Drosophila genetic resource of mutants to study mechanisms underlying human genetic diseases. Cell 159, 200-214.

Yoon, K.J., Song, G., Qian, X., Pan, J., Xu, D., Rho, H.S., Kim, N.S., Habela, C., Zheng, L., Jacob, F., et al. (2017). Zika-virus-encoded NS2A disrupts mammalian cortical neurogenesis by degrading adherens junction proteins. Cell Stem Cell 21, 349-358.e6.
Yuan, L., Huang, X.Y., Liu, Z.Y., Zhang, F., Zhu, X.L., Yu, J.Y., Ji, X., Xu, Y.P., Li, G., Li, C., et al. (2017). A single mutation in the prM protein of Zika virus contributes to fetal microcephaly. Science 358, 933-936.

Zeng, J., Dong, S., Luo, Z., Xie, X., Fu, B., Li, P., Liu, C., Yang, X., Chen, Y., Wang, X., et al. (2020)The Zika Virus Capsid Disrupts Corticogenesis by Suppressing Dicer Activity and miRNA Biogenesis. Cell Stem Cell,

Zhang, X., Xie, X., Xia, H., Zou, J., Huang, L., Popov, V.L., Chen, X., and Shi, P.Y. (2019a). Zika virus NS2A-mediated virion assembly. mBio 10, e02375-19.

Zhang, X., Xie, X., Zou, J., Xia, H., Shan, C., Chen, X., and Shi, P.Y. (2019b). Genetic and biochemical characterizations of Zika virus NS2A protein. Emerg Microb Infect 8, 585-602.

Zhao, B., Yi, G., Du, F., Chuang, Y.C., Vaughan, R.C., Sankaran, B., Kao, C.C., and Li, P. (2017). Structure and function of the Zika virus fulllength NS5 protein. Nat Commun 8, 14762.

Zheng, Y., Liu, Q., Wu, Y., Ma, L., Zhang, Z., Liu, T., Jin, S., She, Y., Li, Y. P., and Cui, J. (2018). Zika virus elicits inflammation to evade antiviral response by cleaving cGAS via NS1-caspase-1 axis. EMBO J 37, e99347.

Zhou, J., Chi, X., Cheng, M., Huang, X., Liu, X., Fan, J., Xu, H., Lin, T., Shi, L., Qin, C., et al. (2019). Zika virus degrades the $\omega-3$ fatty acid transporter Mfsd2a in brain microvascular endothelial cells and impairs lipid homeostasis. Sci Adv 5, eaax7142. 